\title{
Effects of Pharmacist Intervention on Community Control of Hypertension: A Randomized Controlled Trial in Zunyi, China
}

\author{
Ying Li, a,b Guoqin Liu, ${ }^{a}$ Chaojie Liu, ${ }^{c}$ Xianhong Wang, ${ }^{a}$ Yalin Chu, ${ }^{a}$ Xiaoqin Li, ${ }^{a}$ Wenhao Yang, ${ }^{a}$
} Yewei Shen, ${ }^{a}$ Fang Wu, ${ }^{a}$ Wenzhi Zhang ${ }^{d}$

\section{Key Finding}

- The results from this randomized controlled trial of a pharmacist intervention, which included patient education and medication, are encouraging, showing significant short-term (at 3-6 months) improvement in blood pressure control, patient knowledge on hypertension, and medication adherence.

\section{Key Implications}

- Community pharmacists can contribute to better management of chronic conditions, such as hypertension, as part of an interdisciplinary team in an integrated care network to improve the continuity and coordination of patient care.

- The findings shed some new insight into the unique role that pharmacists can play by working in partnership with the health care providers, patients, and other community health workers.

\footnotetext{
a School of Management, Zunyi Medical University, Zunyi, People's Republic of China.

'The Second Affiliated Hospital of Zunyi Medical University, Zunyi, People's Republic of China.

'School of Psychology and Public Health, La Trobe University, Melbourne VIC 3086, Australia.

dDepartment of Pharmacy, Affiliated Hospital of Zunyi Medical University, Zunyi, People's Republic of China.

Correspondence to Guoqin Liu (253660762@qq.com) and Chaojie Liu (c.liu@latrobe.edu.au).
}

\section{ABSTRACT}

Objective: We aimed to test the effects of pharmacist intervention on the community control of hypertension through a comparative randomized controlled trial.

Methods: We recruited adult hypertensive patients with comorbidity or confusion with medication $(n=636)$ from 2 community health centers in Zunyi, China. They were randomly and equally divided into 2 groups. Both groups received the usual care and participated in the community systematic management program of hypertension. Participants in the intervention group were given interventions from pharmacists, including a monthly review of medications, patient education, and medication adjustment advice to medical doctors over 6 months. Participants' blood pressure was assessed at baseline, 3 months, and 6 months. Participants' knowledge and medication adherence were measured using a questionnaire before and after the trial.

Results: Compared to the control group ( $n=298)$, a significantly higher percentage of participants in the intervention group $(n=290)$ had their blood pressure under control 3 months (46.9\% vs. $38.3 \%, P=.034)$ and 6 months $(60.7 \%$ vs. $40.9 \%$, $P<.001)$ after the interventions. Difference-in-differences analyses showed that the pharmacist intervention resulted in an increase in knowledge scores by 12.55 points $(P<.001)$, a decrease in systolic blood pressure by $6.65 \mathrm{mmHg}(P=.001)$, and a decrease in diastolic blood pressure by $7.26 \mathrm{mmHg}$ $(P<.001)$ compared to the controls after adjustment for variations in potential confounding factors. The odds of participants passing the hypertension knowledge tests in the intervention group was 4.45 times those in the control group $(P<.001)$. Similarly, it was found that the intervention group had higher odds of not needing any medication adjustments (adjusted odds ratio $[A O R]=2.75, P<.001)$ and having their blood pressure under control ( $A O R=2.18, P=.002)$ compared to the control group.

Conclusion: It is evident that pharmacist intervention has significant short-term effects on improving the knowledge and medication adherence of hypertensive patients, as well as timely medication adjustments from medical doctors, resulting in lowered blood pressure and an increased control rate. Further studies should explore the long-term sustainability of the effects of community pharmacist intervention.

\section{INTRODUCTION}

$\mathbf{H}$ ypertension is the most common chronic disease and a primary risk factor for cardiovascular and cerebrovascular diseases. Although effective treatment regimens exist for hypertension that can significantly 
reduce mortality and prevent the development of complications, ${ }^{1}$ the control of blood pressure has remained a great challenge. The World Health Organization (WHO) estimated that 1.13 billion people in the world have hypertension. ${ }^{2}$ But more than four-fifths have failed to bring their blood pressure under control, resulting in 10 million deaths every year. ${ }^{3}$ Hypertension also brings substantial financial burdens to the world, which affect low- and middle-income countries (LMICs) disproportionately. About two-thirds of hypertensive patients in the world live in China. ${ }^{2}$ A study found that hypertension treatments account for $6.61 \%$ of the total health expenditure in China. ${ }^{4}$

There are many underlying reasons for the poor control of blood pressure, of which poor knowledge of patients and nonadherence to prescribed therapy are deemed critical contributing factors. ${ }^{5-7}$ It is widely believed that optimizing drug treatment is the key to achieving the successful control of blood pressure. ${ }^{5,8,9}$ A systematic review and meta-analysis showed that $83.7 \%$ of cases of uncontrolled hypertension are a result of nonadherence to drug therapy. ${ }^{7}$ But unfortunately, there is no simple solution. Many efforts have been made to address this issue, ranging from patient education and guidelines on professional practice to technological innovations. ${ }^{10}$ Empirical evidence has highlighted the importance of cross-disciplinary collaboration and partnerships between health care providers and patients. $^{11}$

In recent years, interest has grown in enhancing pharmacist services in primary care for improved management of hypertension. Pharmacist services have shifted focus from ensuring drug supply to providing patient-centered care. Studies conducted in high-income countries, such as the United States and Canada, have demonstrated that pharmacist interventions on hypertension can help achieve remarkable results, ${ }^{12-14}$ including improved adherence to drug therapy, lowered blood pressure, better health outcomes, and savings in relation to medical expenditure. ${ }^{15-20}$ Consequently, the Canadian ${ }^{8,21}$ government has issued guidelines and standards for community pharmacist care for hypertensive patients. As a highly skilled profession, community pharmacists have performed well in monitoring chronic conditions, educating patients, and providing medication consultations to medical doctors and patients. $^{11,15,22}$

However, there is a dearth of evidence on the effectiveness of community pharmacist interventions on hypertension in LMICs. In a systematic review on the impact of interventions by community pharmacists on the control of hypertension, Cheema et al. ${ }^{23}$ identified only 5 of 16 randomized controlled trials conducted in LMICs. There is a consensus that community-oriented primary care is the most appropriate setting for managing hypertension, which requires continuous and coordinated care. ${ }^{24}$ In high-income countries, pharmacists are usually more easily accessible in the community than medical doctors, giving community pharmacists an advantage in managing hypertension. But this is not necessarily the case in LMICs where a shortage of properly trained pharmacists is common. ${ }^{25}$

This study fills the gap in the literature by testing the impact of pharmacist interventions on hypertension in primary care facilities in China. Since 2009, the Chinese government has increased its investment in primary care dramatically. A total of 9,352 (in 2018) community health centers were developed over a short period, covering the entire population. ${ }^{26}$ In line with the international evidence, community health care services have been shown to serve as a major force in managing chronic conditions. However, workforce development in community health care services has been focused on general practice and community nursing. Little attention, if any, has been paid to the development of community pharmacists although all community health care services dispense medications. The outcome of the primary care reform in China has attracted serious debate. ${ }^{27}$ It appears that the management programs for chronic diseases have been far from successful. In 2018 , only $30 \%$ of hypertensive patients received antihypertension treatments, ${ }^{28}$ well below the level $(40 \%-80 \%)$ of high-income countries such as Canada, Australia, the United Kingdom, the United States, and Germany. ${ }^{29}$ The community management of chronic diseases is delivered in China free of charge by a general practice team as part of the essential public health service package. ${ }^{30,31}$ High workloads of the general practice team and a lack of attention to medication adherence have often been blamed for the unsatisfactory outcome. ${ }^{4,32,33}$ Indeed, community health care services have been struggling to attract medical doctors. In China, about 1.53 million medical doctors (2.5 per 1,000 population) work in primary care, accounting for $39 \%$ of the medical workforce. ${ }^{34}$ Adding to the complexity is the low qualifications of primary care medical workers $\left(31 \%\right.$ do not have a medical degree), ${ }^{35}$ the high prevalence of irrational prescriptions, ${ }^{28}$ and the self-medication of consumers. ${ }^{36}$ As a result, there have been increasing calls recently from

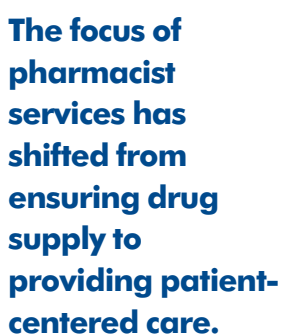


both researchers and policy makers to explore the role of community pharmacists.

\section{MATERIALS AND METHODS}

\section{Study Setting}

A comparative randomized controlled trial was conducted in 2 urban community health centers in the Zunyi municipality of Guizhou province in China. The trial was registered with the Chinese Clinical Trials Registry (registration number: ChiCTR1900028368).

Zunyi has a population of more than 6 million. In 2018 , it had an average gross domestic product (GDP) of US\$5,656 per capita, ${ }^{37}$ about half of the national average in mainland China. ${ }^{38}$ According to the World Bank, Zunyi is ranked at the lower end of middle-income economies. ${ }^{39}$

Community health services were developed in China as a hub for the delivery of primary health care services, covering essential medical services, disease prevention and control, care for vulnerable populations, rehabilitation, family planning, health education, and health promotion. Each community health center was designated to cover a residential community ranging in number from 20,000 to 60,000 people. Zunyi has 19 urban community health centers. ${ }^{37}$ In 2018, a total of 822,600 patients sought medical attention from these health centers. ${ }^{40}$

Two medium-sized urban community health centers were selected from the Huichuan district, the economic, political, and cultural center of Zunyi. Huichuan was ranked 48 among the 219 economic and technological development zones in China. ${ }^{41} \mathrm{~A}$ total of 6,500 hypertensive patients registered with the 2 centers over the past 9 years.

\section{Study Participants}

Eligible participants of this study were those aged 18 years or older who had a confirmed diagnosis of hypertension. They had enrolled in the community systematic management program for hypertension and received relevant services. The participants also had to meet at least 1 of the following criteria: (1) taking antihypertensive medications; (2) having coexisting chronic conditions such as diabetes; (3) reporting confusion with their own medication regimen; or (4) missing medicines frequently. Pregnant and lactating women and patients with recorded cognitive impairments were excluded from this study.

Eligible patients who sought medical attention for hypertension from the 2 community health centers from January to April 2018 were invited by their doctors to participate in this study. In total, 746 were invited and 110 rejected. This resulted in a sample size of 636 participants, who were randomly allocated into the control and intervention groups equally. Eventually, 28 participants dropped out of the intervention group, compared with 20 in the control group (Figure 1).

The final sample size $(n=588)$ included for data analysis was large enough to enable the detection of a $20 \%$ difference in the percentage of participants with blood pressure under control between the 2 groups. A national survey showed that about $38 \%$ of hypertensive patients had their blood pressure well controlled, ${ }^{42}$ but the government aimed for a $60 \%$ goal. ${ }^{43}$ A sample of 160 participants in each group would be required to detect the significance of such an achievement at $\alpha=0.05$ and $\beta=0.80$ according to the formula below:

$$
\begin{aligned}
& N= \\
& \frac{\left[Z_{\alpha} \sqrt{2 P(1-P)}+Z_{\beta} \sqrt{P_{c}\left(1-P_{c}\right)+P_{e}\left(1-P_{e}\right)}\right]^{2}}{\left(P_{c}-P_{e}\right)^{2}}
\end{aligned}
$$

Where $\mathrm{Z}_{\alpha}$ for $\alpha$ level is the corresponding standard normal, $\mathrm{Z}_{\beta}$ for $\beta$ level is the corresponding standard normal, $\mathrm{N}$ is the required sample size for each group, and $\mathrm{P}_{\mathrm{c}}$ and $\mathrm{P}_{\mathrm{e}}$ represent the percentage of participants with their blood pressure under control in the control and the intervention groups, respectively; $\mathrm{P}=\left(\mathrm{P}_{\mathrm{c}}+\mathrm{P}_{\mathrm{e}}\right) / 2$.

The sample size was also large enough to enable detection of a very small effect size $(0.1)$ for the continuous indicators (knowledge and medication adherence scores) while $\alpha$ being set at 0.05 and statistical power $(1-\beta)$ being set at $0.8 .{ }^{44}$

\section{Randomization}

The random allocation of study participants was conducted by the researchers who were not involved in the implementation of the trial. The eligible patients were sorted in order according to the time when they agreed to participate in the trial. They were divided into 2 groups using a table of random numbers, which involved 3 steps. First, a starting point was randomly identified in the table. Then a backward or forward direction was randomly determined to retrieve 636 random numbers from the identified starting point of the table. Finally, the random numbers were mapped with the participants in sequence, which were then reordered from small to large. The first 318 patients 
FIGURE 1. Flow Chart of Participant Recruitment in a Randomized Controlled Trial on Effect of Pharmacist Interventions on Management of Hypertension, China

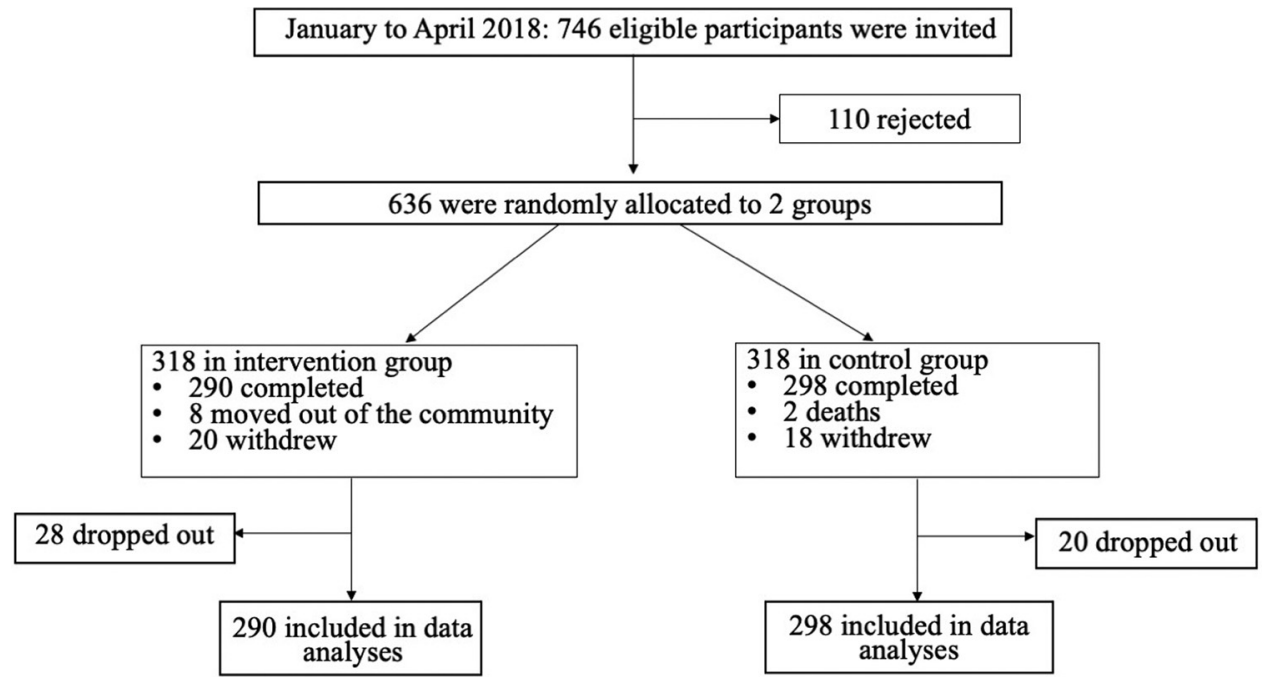

with smaller numbers were assigned to the control group, while the rest were assigned to the intervention group.

\section{Trial Protocol}

The trial started in June 2018 and lasted for 6 months.

All the participants received the usual care and continued their community systematic management program delivered by a team comprising general practitioners, nurses, and public health workers. The systematic management program for hypertension was part of the National Essential Public Health Package being offered to all eligible community residents for free. Once every 3 months, follow-up with hypertensive patients was conducted at outpatient appointments, telephone interviews, and home visits by monitoring patients' blood pressure and heart rate; changes in symptoms, comorbidities, and critical complications; risk factors (such as body mass index, smoking, drinking, salt intake, and physical exercise); and medication compliance. The patients identified with poorly controlled conditions were supposed to be given further medical advice or referred to a specialist clinic if needed and followed up again 2 weeks later. The routine follow-up arrangement was complemented with an annual physical examination, including blood tests and electrocardiograms. ${ }^{31}$ In addition to the community systematic management program, patients also had the freedom to bypass community health services to seek medical attention from hospitals covered by social health insurance programs. By 2011, almost all Chinese citizens had been covered by social health insurance policies, albeit with varied benefit entitlements. $^{45}$

This trial tested the effectiveness of additional interventions involving pharmacists on the control of blood pressure. Five registered pharmacists were recruited from local tertiary hospitals (in China, very few, if any, properly trained registered pharmacists work in community health services). All of them had a master's degree and had engaged in clinical practice for more than 8 years. They showed a strong willingness to participate in the trial and maintained high levels of commitment throughout the trial. The pharmacists conducted a medication review for each patient in the intervention group based on the data collected through the baseline survey. A medication adjustment plan, if needed, was then developed considering the safety, effectiveness, and adherence barriers. The medication adjustment plan was further reviewed and fine-tuned monthly by the pharmacists based on a chart report. The monthly medication chart was developed by the assistants (medical students) of the pharmacists through outpatient appointments, home visits, or telephone interviews, during which they recorded patient uptake and adherence to the prescriptions 
(dosage, timing, and frequency of medicines), selfrecorded blood pressure (at least weekly), and patients' main symptom complaints. If necessary, pharmacists conducted additional telephone interviews to better understand the situation of the patients (such as those with missing information). Interviews lasted about 6-10 minutes. In total, no more than 5 additional phone interviews were conducted each month. There was not a fixed arrangement between a pharmacist and the patients. A patient might receive the intervention from different pharmacists (or/and their assistants) at different times.

The pharmacists informed both patients and their general practitioners of the proposed medication adjustments, but general practitioners were responsible for advising the patients about any medication changes. Meanwhile, the pharmacists also developed an individualized education plan for each of the patients with risk behaviors. The plan was conveyed to the patient orally by the pharmacists or their assistants. Three months after the start of the intervention, the pharmacists delivered a group presentation in each community health center for the participants in the intervention group. More than $80 \%$ of the patients in the intervention group attended the group presentations.

\section{Measurements}

The intervention effect was measured by 3 indicators:

1. Blood pressure: Blood pressure was measured by a calibrated sphygmomanometer (Omron HBP-1300 electronic sphygmomanometer) and recorded as systolic/diastolic mmHg. Each participant was measured 3 times with a 5-minute interval. An average reading was recorded. For most patients including those with diabetes or/and chronic kidney diseases, a reading below $140 / 90 \mathrm{mmHg}$ was deemed normal and achievable. But a reduction of blood pressure to lower than $150 / 90 \mathrm{mmHg}$ for those aged over 65 years with no complications was also considered acceptable. ${ }^{31}$

2. Hypertension knowledge: Patient knowledge of hypertension was measured by a scale developed by the research team. The scale was adapted from 2 existing instruments ${ }^{23,46}$ and guided by the 2017 Chinese National Guidelines for Prevention and Control of Hypertension in Primary Care. ${ }^{31}$ The scale contained 10 items, measuring the definition, risk factors, potential complications, and available interventional strategies of hypertension.
A correct answer was given a score of 1 , otherwise 0 . Four of the items contained more than 1 correct answer. This resulted in a summed score ranging from 0 to 32. The summed score was transformed into a percentage score, with a higher score indicating higher knowledge and $>60 \%$ being considered "pass." Consultations were sought from 10 experts specialized in medicine, pharmacy, and public health on the content validity of the scale. The scale had a Cronbach's $\alpha$ of 0.919 , indicating excellent internal consistency.

3. Medication adherence: Medication adherence refers to the degree to which a patient implements the medication treatment regimen. It was assessed using a validated 8 item scale developed by Morisky (MMAS-8) ${ }^{47}$ Respondents were asked to answer "yes" (1) or "no" (0) to the first 7 items. The last item was rated on a 5-point Likert scale ranging from 0 to 1 . The scores were then summed, with a higher score indicating higher adherence. A score lower than 6 was deemed low adherence, while a score of 8 was considered high adherence. The MMAS- 8 had a Cronbach's $\alpha$ of 0.786 in this study, indicating good internal consistency. In addition, the percentage of participants taking antihypertensive medications regularly and the percentage of participants with a treatment regimen with no need for adjustment were also calculated.

\section{Data Collection}

The blood pressure of the participants was recorded before the trial (baseline, June 2018) and 3 and 6 months after commencement of the trial. Data about hypertension knowledge and medication adherence were collected twice through a questionnaire survey: once at the baseline and again at the end of the trial, which also included information about the sociodemographic characteristics (age, gender, and education), risk factors (smoking, drinking, exercise, and salt intake), duration of diagnosed hypertension, and coexisting chronic conditions of the respondents.

Participating pharmacists' assistants conducted household visits, measured blood pressure, and administered the questionnaire through face-to-face interviews. All the assistants were postgraduate or undergraduate medical students and were trained and assessed against the 2017 National Guidelines for Prevention and Control of Hypertension in Primary Care $^{31}$ before interacting with patients. A quality control officer examined logical errors embedded in the returned questionnaires. 


\section{Data Analysis}

This was a comparative trial. Although it was not possible to conduct a blinded trial, data were not analyzed until the trial ended. Data were input into Epidata 3.1 and analyzed using Stata version 15.0. A $P$ value of less than .05 was considered statistically significant.

The number and percentage of participants with different sociodemographic characteristics were described. Differences in the outcome indicators between the intervention and control groups were compared using independent sample t-tests for the continuous measurements or Chisquare tests for the categorical measurements. Paired t-tests or Chi-square tests were performed to examine changes in the outcome indicators over time. Difference-in-differences (DID) analyses were performed to provide additional evidence on the effects of pharmacist intervention based on a linear regression model (for continuous measurements) or logistic regression model (for categorical measurements), adjusting for potential variations in confounding factors including age, gender, education, lifestyle (smoking, drinking, salt intake, and physical exercises), duration of hypertension, and coexisting chronic conditions:

$$
\begin{aligned}
y_{\text {it }}\left(\text { or } \operatorname{Logit} y_{\mathrm{it}}\right)= & \beta_{0}+\beta_{1} \cdot \operatorname{group}_{\mathrm{it}}+\beta_{2} \cdot \text { time }_{\mathrm{it}} \\
& +\beta_{3} \cdot \text { group }_{\mathrm{it}} \cdot \text { time }_{\mathrm{it}}+\varphi X_{\mathrm{it}} \\
& +\varepsilon_{\mathrm{it}}
\end{aligned}
$$

Where $y$ is the outcome indicator (dependent variable), i represents each individual, $t$ is different times; time is a dummy variable with 0 indicating pre-trial and 1 indicating posttrial; group indicates the group allocation of participants $(0=$ control, $\mathrm{l}=$ intervention $) ; \mathrm{X}$ represents all the control variables; $\varepsilon$ is a random error. The interaction effect between group and time (group multiplied by time) detects the effect of the intervention. An enter approach was adopted.

\section{Ethics Approval}

The study protocol was approved by the Ethics Committee of Zunyi Medical University. The trial was registered with the Chinese Clinical Trials Registry (registration number: ChiCTR1900028368).

Before the trial and data collection, the study nature, objectives, and details were explained to the potential participants. Written informed consent was obtained from those who were willing to participate in the study. The participants were informed about their right to withdraw from the study, which would not result in any negative consequences on their services and treatment. They were assured of confidentiality.

\section{RESULTS}

\section{Participant Baseline Characteristics}

A total of 588 patients completed this study: 290 in the intervention group and 298 in the control group. The study participants had an average age of 65.98 years (standard deviation $[S D]=9.48$ ). More than half $(57.31 \%)$ were female. The majority $(52.72 \%)$ had only completed primary school education. About $18.71 \%$ were smoking, 26.02\% were drinking, and $17.86 \%$ had heavy salt intake $\left(>5 \mathrm{~g} \mathrm{a} \mathrm{day}^{48}\right)$ at the time of the baseline survey. Approximately $86.73 \%$ reported regular daily exercise of moderate intensity, such as walking, housework, sports, and recreational activities (according to the WHO, these activities consume about 3-6 metabolic equivalent oxygen). ${ }^{49}$ About one-fifth $(19.39 \%)$ of the participants reported coexisting chronic conditions. More than $30 \%$ of the participants had been living with hypertension for 10 or more years. But less than $10 \%$ had their blood pressure under control. There were no significant differences in the baseline characteristics between the intervention and control groups, except for age. The intervention group had a higher percentage of elderly (aged $\geq 65$ years) participants, about 2.55 years older than the control group $(P<.001)$ (Table 1$)$.

\section{Blood Pressure}

Control of blood pressure improved over time in both groups $(P<.005)$. However, a significantly higher percentage of participants in the intervention group had their blood pressure under control (<140/90 mmHg or $<150 / 90 \mathrm{mmHg}$ for those aged over 65 years with no complications) at the end of the trial compared with the control group $(60.7 \%$ vs. $40.9 \%, P<.001)$, despite a lack of difference in the baseline (Table 2).

The effects of the intervention became significant 3 months after the trial $(46.9 \%$ vs. $38.3 \%$, $P=.034$ ) and continued until the end of the trial (Figure 2).

Although there were no significant differences at baseline in systolic blood pressure (SBP) $(P=.147)$ and diastolic blood pressure (DBP) $(P=.713)$ readings between the intervention and control groups, significant differences appeared in 
TABLE 1. Baseline Characteristics of Study Participants in a Randomized Controlled Trial on Effect of Pharmacist Interventions on Management of Hypertension, China

\begin{tabular}{|c|c|c|}
\hline & $\begin{array}{c}\text { Intervention }(n=290) \\
\text { No. }(\%)\end{array}$ & $\begin{array}{c}\text { Control }(n=298) \\
\text { No. }(\%)\end{array}$ \\
\hline \multicolumn{3}{|l|}{ Gender } \\
\hline Male & $115(39.7)$ & $136(45.6)$ \\
\hline Female & $175(60.3)$ & $162(54.4)$ \\
\hline \multicolumn{3}{|l|}{ Age, years } \\
\hline$<65$ & $81(27.9)$ & $136(45.6)$ \\
\hline $65-79$ & $197(67.9)$ & $139(46.6)$ \\
\hline$\geq 80$ & $12(4.1)$ & $23(7.7)$ \\
\hline \multicolumn{3}{|l|}{ Education } \\
\hline Primary school & $160(55.2)$ & $150(50.3)$ \\
\hline Middle school & $81(27.9)$ & 83 (27.9) \\
\hline High school & $34(11.7)$ & $51(17.1)$ \\
\hline University & $15(5.2)$ & $14(4.7)$ \\
\hline \multicolumn{3}{|l|}{ Smoking } \\
\hline No & $243(83.8)$ & $235(78.9)$ \\
\hline Yes & $47(16.2)$ & $63(21.1)$ \\
\hline \multicolumn{3}{|l|}{ Drinking } \\
\hline No & $223(76.9)$ & $212(71.1)$ \\
\hline Yes & $67(23.1)$ & $86(28.9)$ \\
\hline \multicolumn{3}{|c|}{ Regular daily exercise } \\
\hline No & $33(11.4)$ & $45(15.1)$ \\
\hline Yes & $257(88.6)$ & $253(84.9)$ \\
\hline \multicolumn{3}{|l|}{ Salt intake } \\
\hline Light & $113(39.0)$ & $105(35.2)$ \\
\hline Medium & $123(42.4)$ & $142(47.7)$ \\
\hline Heavy & $54(18.6)$ & $51(17.1)$ \\
\hline \multicolumn{3}{|c|}{ Years of living with hypertension } \\
\hline$<10$ & $195(67.2)$ & $210(70.5)$ \\
\hline $11-20$ & $75(25.9)$ & $71(23.8)$ \\
\hline$>20$ & $78(6.2)$ & $15(5.0)$ \\
\hline Not sure & $2(0.7)$ & $2(0.7)$ \\
\hline \multicolumn{3}{|c|}{ Other chronic conditions } \\
\hline Yes & $64(22.1)$ & $50(16.8)$ \\
\hline No & $226(77.9)$ & $248(83.2)$ \\
\hline \multicolumn{3}{|c|}{ Blood pressure $(\mathrm{mmHg})^{a}$} \\
\hline$<120 / 80$ & $10(3.4)$ & $6(2.0)$ \\
\hline $120-139 / 80-89$ & $62(21.4)$ & $72(24.2)$ \\
\hline $140-159 / 90-99$ & $118(40.7)$ & $141(47.3)$ \\
\hline
\end{tabular}


TABLE 1. Continued

\begin{tabular}{lcc}
\hline & $\begin{array}{c}\text { Intervention }(n=290) \\
\text { No. }(\%)\end{array}$ & $\begin{array}{c}\text { Control }(n=298) \\
\text { No. }(\%)\end{array}$ \\
\hline $160-179 / 100-109$ & $72(24.8)$ & $63(21.1)$ \\
$\geq 180 / 110$ & $28(9.7)$ & $16(5.4)$
\end{tabular}

aBlood pressure was presented as SBP/DBP and the higher grade prevails.

TABLE 2. Study Participants With Blood Pressure Under Control in a Randomized Controlled Trial on Effect of Pharmacist Interventions on Management of Hypertension, China

\begin{tabular}{lccr}
\hline Time & Intervention, No. (\%) & Control, No. (\%) & P Value \\
\hline Baseline & $95(32.8 \%)$ & $92(30.9 \%)$ & .62 \\
\hline Third month & $136(46.9 \%)$ & $114(38.3 \%)$ & .03 \\
\hline Sixth month & $176(60.7 \%)$ & $122(40.9 \%)$ & $<.001$ \\
\hline$P$ value & $<.001$ & .03 &
\end{tabular}

FIGURE 2. Changes in Systolic and Diastolic Blood Pressure Readings ( $\mathrm{mmHg}$ ) Among Participants in a Randomized Controlled Trial on Effect of Pharmacist Interventions on Management of Hypertension, China

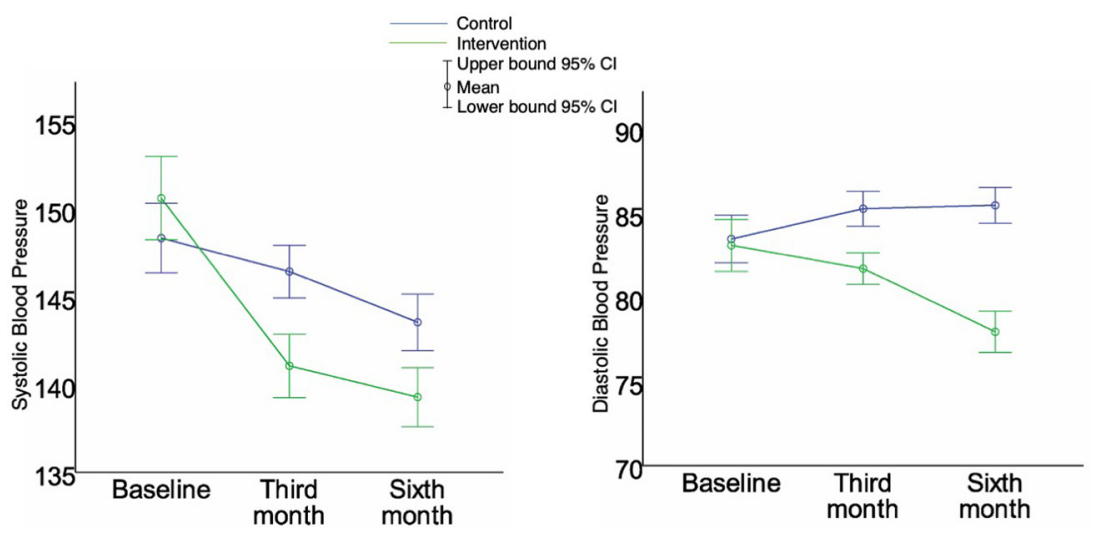

both SBP $(P<.001)$ and DBP $(P<.001) 3$ months after the trial. The gaps between the 2 groups remained at the end of the trial $(P<.001)$ (Table $3)$. The intervention group ended up with significantly lower SBP and DBP readings than the control group. Both groups experienced a drop in SBP over time, but to a greater extent in the intervention group. The DBP reading declined in the intervention group, compared with a slight rise in the control group.

\section{Hypertension Knowledge}

Hypertension knowledge scores increased over time in both groups $(P<.001)$. However, significantly higher knowledge scores were found in the intervention group at the end of the trial compared with the control group (77.46 \pm 19.33 vs. $61.00 \pm 26.98, P<.001)$, despite a lack of difference in the baseline. At the baseline, about $37 \%$ of participants in the intervention group and $32 \%$ in the control group passed (scored over 60) the knowledge 
TABLE 3. Blood Pressure Readings of Study Participants in a Randomized Controlled Trial on Effect of Pharmacist Interventions on Management of Hypertension, China

\begin{tabular}{llccr}
\hline & & Intervention, mean \pm SD & Control, mean \pm SD & PValue \\
\hline Systolic blood pressure & Baseline & $150.61 \pm 20.44$ & $148.34 \pm 17.33$ & .15 \\
\cline { 2 - 5 } & Third month & $141.07 \pm 15.52$ & $146.44 \pm 13.24$ & $<.001$ \\
\cline { 2 - 5 } & Sixth month & $139.29 \pm 14.53$ & $143.54 \pm 14.12$ & $<.001$ \\
\hline & $P$ Value & $<.001$ & $<.001$ & .72 \\
\hline Diastolic blood pressure & Baseline & $83.09 \pm 13.38$ & $83.47 \pm 12.38$ & $<.001$ \\
\cline { 2 - 5 } & Third month & $81.70 \pm 8.21$ & $85.27 \pm 9.11$ & $<.001$ \\
\cline { 2 - 5 } & Sixth month & $77.94 \pm 10.51$ & $85.47 \pm 9.29$ & .04 \\
\hline
\end{tabular}

Abbreviation: SD, standard deviation.

TABLE 4. Hypertension Knowledge of Study Participants in a Randomized Controlled Trial on Effect of Pharmacist Interventions on Management of Hypertension, China

\begin{tabular}{|c|c|c|c|c|c|c|}
\hline & \multicolumn{3}{|c|}{ Knowledge Score, mean \pm SD } & \multicolumn{3}{|c|}{ Participants with $\geq 60$ Score, No. (\%) } \\
\hline & Intervention & Control & PValue & Intervention & Control & PValue \\
\hline Sixth month & $77.46 \pm 19.33$ & $61.00 \pm 26.98$ & $<.001$ & $244(84.10 \%)$ & $151(50.70 \%)$ & $<.001$ \\
\hline PValue & $<.001$ & $<.001$ & & $<.001$ & $<.001$ & \\
\hline
\end{tabular}

Abbreviation: SD, standard deviation.

tests $(P=.2)$. This increased to $84 \%$ and $51 \%$ in the intervention group and the control group, respectively, at the end of the trial $(P<.001)$ (Table 4$)$.

\section{Medication Adherence}

Significant differences in patient adherence to medications were found at the baseline between the intervention and control groups. Participants in the intervention group were more likely to take medications regularly $(P<.001)$, adhere to prescriptions $(P<.001)$, and have no need to adjust their treatment regimen $(P=.02)$. Such differences remained at the conclusion of the trial $(P<.001)$ although participants in both groups experienced significant improvement $(P<.05)$ (Table 5).

\section{Results of DID Analyses}

The DID analyses confirmed that the intervention improved control of blood pressure significantly in terms of the 3 outcome indicators after adjustments for potential variations in confounding factors (Table 6). The intervention resulted in an increase in knowledge scores by 12.55 points $(P<.001)$, a decrease in SBP readings by 6.65 $\mathrm{mmHg}(P=.001)$, and a decrease in DBP readings by $7.26 \mathrm{mmHg}(P<.001)$ in comparison with the controls. The odds of participants passing hypertension knowledge tests in the intervention group was 4.45 times those in the control group $(P<.001)$. Similarly, it was found that the intervention group had higher odds of not needing any adjustments of the treatment regimen (AOR=2.75, $P<.001$ ) and having their blood pressure under control $(\mathrm{AOR}=2.18, P=.002)$ compared with the control group.

\section{DISCUSSION}

This study shows that community pharmacist interventions improve knowledge and medication 
TABLE 5. Antihypertensive Drug Treatment of Study Participants in a Randomized Controlled Trial on Effect of Pharmacist Interventions on Management of Hypertension, China

\begin{tabular}{|c|c|c|c|c|c|c|c|c|c|}
\hline \multirow{2}{*}{$\begin{array}{l}\text { Antihypertensive } \\
\text { Treatment }\end{array}$} & & \multicolumn{3}{|c|}{ Intervention, No. (\%) } & \multicolumn{3}{|c|}{ Control, No. (\%) } & \multicolumn{2}{|c|}{$\begin{array}{c}\text { P Value for } \\
\text { Group Difference }\end{array}$} \\
\hline & & Baseline & Sixth Month & P Value & Baseline & Sixth Month & PValue & Baseline & Sixth Month \\
\hline \multirow{2}{*}{$\begin{array}{l}\text { Administration of } \\
\text { medications }\end{array}$} & Regularly & $234(80.7 \%)$ & 277 (95.5\%) & \multirow[t]{2}{*}{$<.001$} & $191(64.1 \%)$ & $210(70.5 \%)$ & \multirow[t]{2}{*}{$<.001$} & \multirow[t]{2}{*}{$<.001$} & \multirow[t]{2}{*}{$<.001$} \\
\hline & Intermittently & $43(14.8 \%)$ & $13(4.5 \%)$ & & $48(16.1 \%)$ & $16(5.4 \%)$ & & & \\
\hline \multirow{2}{*}{$\begin{array}{l}\text { Medication adher- } \\
\text { ence score }\end{array}$} & Medium (6-7) & $80(27.6 \%)$ & $14(4.8 \%)$ & \multirow{2}{*}{$<.001$} & $61(20.5 \%)$ & $18(6.0 \%)$ & \multirow{2}{*}{$<.001$} & \multirow{2}{*}{$<.001$} & \multirow{2}{*}{$<.001$} \\
\hline & High (=8) & 147 (50.7\%) & $263(90.7 \%)$ & & $126(42.3 \%)$ & $191(64.1 \%)$ & & & \\
\hline \multirow{2}{*}{$\begin{array}{l}\text { Need for adjust- } \\
\text { ment on treatment } \\
\text { regimen }\end{array}$} & No & 93 (32.1\%) & $184(63.4 \%)$ & \multirow[t]{2}{*}{$<.001$} & $70(23.5 \%)$ & $91(30.5 \%)$ & \multirow[t]{2}{*}{.03} & \multirow[t]{2}{*}{.02} & \multirow[t]{2}{*}{$<.001$} \\
\hline & Yes & 197 (67.9\%) & $106(36.6 \%)$ & & $228(76.5 \%)$ & $207(69.5 \%)$ & & & \\
\hline
\end{tabular}

TABLE 6. Difference-in-Differences Analyses on the Effects of Pharmacist Interventions on Study Participants' Blood Pressure

Outcome Indicator

\begin{tabular}{|c|c|c|c|c|c|c|}
\hline \multirow[b]{2}{*}{ Linear Regression Model for Continuous Measurement } & \multicolumn{3}{|c|}{ Unadjusted $\beta$ Coefficient } & \multicolumn{3}{|c|}{ Adjusted $\beta$ Coefficient ${ }^{a}$} \\
\hline & Estimate & $(95 \% \mathrm{Cl})$ & P Value & Estimate & $(95 \% \mathrm{Cl})$ & P Value \\
\hline Hypertension knowledge score & 12.74 & $(12.56,12.91)$ & $<.001$ & 12.55 & $(12.38,12.71)$ & $<.001$ \\
\hline Systolic blood pressure reading & -6.52 & $(-6.63,-6.41)$ & .001 & -6.65 & $(-10.39,-2.91)$ & .001 \\
\hline Diastolic blood pressure reading & -7.15 & $(-7.23,-7.08)$ & $<.001$ & -7.26 & $(-9.27,-4.80)$ & $<.001$ \\
\hline Logistic Regression Model for Categorical Measurement & Estimate & $(95 \% \mathrm{Cl})$ & PValue & Estimate & $(95 \% \mathrm{Cl})$ & $P$ Value \\
\hline$\%$ of patients with $\geq 60 \%$ hypertension knowledge score & 4.13 & $(2.47,6.94)$ & $<.001$ & 4.45 & $(2.60,7.59)$ & $<.001$ \\
\hline$\%$ of patients with no need for adjustment on treatment regimen & 2.57 & $(1.56,4.24)$ & $<.001$ & 2.75 & $(1.64,4.60)$ & $<.001$ \\
\hline$\%$ of patients with blood pressure under control & 2.04 & $(0.35,0.57)$ & .003 & 2.18 & $(1.33,3.58)$ & .002 \\
\hline
\end{tabular}

Abbreviation: $\mathrm{Cl}$, confidence interval.

${ }^{a}$ Adjustment for variations in gender, age, education, salt intake, smoking, drinking, physical exercise, duration of hypertension, and other chronic conditions

adherence of hypertensive patients in the short term, which may have the potential to serve as an effective strategy for achieving the target control rate set by the Chinese government. Six months after the trial, $60.7 \%$ of the participants who received pharmacist interventions had their blood pressure under control, already exceeding the government target. In contrast, only $40.9 \%$ of the participants in the control group had their blood pressure under control, 19 percentage points below the governmental target. The marginal contribution of pharmacist interventions is high according to the DID analyses-more than doubling the odds of having blood pressure under control. This result is consistent with the findings of previous studies, although most were conducted in high-income countries. ${ }^{50-53}$ It shed some light on the hope in LMICs that hypertension can be controlled through community pharmacist intervention programs. Santschi and Colosimo ${ }^{54}$ concluded in a meta-analysis that better control of blood pressure can be achieved if pharmacists 


Six months after
the trial, $60.7 \%$ of
the participants
who received
pharmacist
interventions had
their blood
pressure under
control, already
exceeding the
government
target.

take a leading role in community interventions and engage in teamwork monthly. The intervention protocol tested in this trial aligns well with their proposal. However, our study did not test the long-term effect of the intervention, which warrants further studies.

The study provides new insight into the potential role of pharmacists in the community control of hypertension in the Chinese health system context. It proved that pharmacist services in primary care can bring additional benefits, boosting the achievements of the existing medical doctor-led management program for hypertension. Two major mechanisms are likely to have enabled such an outcome.

First, pharmacists, as a profession that specializes in medications, are well positioned to educate patients on the appropriate use of medicines, which can be done through public education and individualized consultation services. This study adopted both approaches and showed that the knowledge score of the participants who received pharmacist interventions increased by 30 points, compared to less than 20 of those in the control group. This resulted in over $80 \%$ of participants passing the knowledge tests in the intervention group, more than thirty percentage points higher than that in the control group. There is evidence that the knowledge improvement had translated into better medication adherence as indicated by the higher increase in the percentage of participants in the intervention group taking regular medications and obtaining high medication adherence scores. International evidence shows that knowledge is indeed critical in helping hypertensive patients to better understand their conditions and adopt appropriate actions to control blood pressure. ${ }^{55-57}$ According to Amer and Nazir, ${ }^{55}$ pharmacists can help hypertensive patients reduce their concerns over the long-term use of medications, one of the major reasons for the intermittent use and ignorance of medications. It is worth noting that there was still space for further improvement of knowledge in the study participants when the trial concluded. Future studies should explore the knowledge attitudes belief behavior chain reactions of hypertensive patients. $^{58-59}$

Second, pharmacists can assist medical doctors to assess and adjust medication regimens for hypertension. This study revealed that the need for the adjustment of drug therapy was very high before the commencement of the trial: $67.9 \%$ for the intervention group and $76.5 \%$ for the control group. This is not surprising given the low qualification profile of general practitioners in China. There is strong evidence suggesting that the timely adjustment of drug therapy is critical for the control of blood pressure. ${ }^{60}$ This study showed that the need for medication adjustments in the participants in the intervention group dropped from $67.9 \%$ to $30.5 \%$, compared with a slight decline in the control group from $76.5 \%$ to $69.5 \%$. The pharmacist interventions contributed to a more than doubling of the odds of not needing any medication adjustments in comparison with the control group. Unlike Hirsch's trial, ${ }^{22}$ this study did not allow pharmacists to initiate, adjust, or discontinue antihypertensive drug therapy independently. All changes had to be made on the advice of the general practitioners. In China, pharmacists have no prescription rights. They play an auxiliary role with a focus on dispensing prescribed medicines, which is not unique to China. ${ }^{61-62}$ Despite their restricted role, pharmacist interventions in this study achieved a similar effect size on the control of blood pressure compared to that in Hirsch's trial. ${ }^{22}$ Although we did not explore the underlying mechanism of the effect, it is clear that pharmacists could contribute to better management of hypertension. The possible reasons must be discussed in the context of the Chinese health system. There have been serious concerns in China that medical doctors are often overloaded and have failed to dedicate sufficient time to communicate with patients, let alone to engage patients. ${ }^{63}$ Clinical pharmacists played a bridging role in this trial. They not only communicated with and educated the patients but also liaised with medical doctors for better management of patients' blood pressure.

It is important to note that as in many other LMICs, pharmacists in China are in short supply. Their functions are complementary to those of medical doctors, nurses, and public health workers. This study is by no means suggesting that pharmacists should bridge the shortfall of general practitioners in China. Pharmacist interventions have to be considered as an integral part of interdisciplinary team efforts for the community control of hypertension. ${ }^{9,64-67}$ In some countries, community pharmacists have been designated specific roles in managing chronic diseases. ${ }^{23,64,68,69}$ But China has yet to do so. By the end of October 2019, there were a total of 509,374 registered pharmacists in China, which is the equivalent of 3.7 pharmacists per 10,000 population. ${ }^{70}$ This level is very low compared to other countries. According to 
the World Federation of Pharmacists, 6.2 pharmacists per 10,000 population are recommended, although developed countries such as Australia and Canada have far more (10-20) pharmacists per 10,000 population. ${ }^{71}$ The gap in the supply of pharmacists is even greater than that of medical doctors.

A systems approach is needed to maximize the role and functions of pharmacists, in particular, to manage chronic diseases. The distribution of registered pharmacists in China is currently heavily concentrated in large metropolitan areas and large institutions. Very few, if any, pharmacists work in community health services. Although hospital pharmacists have started to increase their involvement in ward rounds and medication consultations, their main role is still dispensing prescribed medicines. In 2017, medication therapy management was written into the revised service specification for registered pharmacists for the first time in China. ${ }^{72}$ This signals a shift toward "patientcentered" pharmacist services. After many years, the Chinese government has started to call for an accelerated transformation of pharmaceutical care. $^{73}$ This provides an opportunity to explore how pharmacist services can be incorporated into the most recent development of the integrated care network, in which large hospitals are encouraged to establish partnerships with primary care institutions to improve the continuity and coordination of patient care. ${ }^{74-75}$ This study recruited 5 hospital pharmacists to lead the pharmacist interventions, but we do not expect community health services to be able to employ registered pharmacists in the short term. Instead, we envisage a situation where community health services appoint and train some staff (not necessarily registered pharmacists) to serve as assistants to hospital pharmacists. Such an arrangement fits well into the mission and scope of integrated care networks in China.

The pharmacist education system in China needs to be reformed. University degree programs should place greater emphasis on patient-centered services to better prepare students for the expanded role of registered pharmacists. Meanwhile, large numbers of pharmacy workers (or pharmacist assistants as labeled in this study) need vocational and continuing education to fulfill a function beyond dispensing/sales of medicines. Community health services can also offer placement opportunities for medical and pharmacy students in senior years or at postgraduate levels to enhance their competency in managing chronic diseases through drug therapy. Serious challenges lie ahead. These changes not only require strong funding and policy support but also require recognition of the new identity of the pharmacist profession. ${ }^{5,13}$

To the best of our knowledge, this is the first randomized controlled trial in China testing the effects of community pharmacist interventions on the control of hypertension. We followed a strict protocol for this randomized control trial. The study participants were randomly selected and highly diversified, which indicates a potential for the results to be generalized to other groups of patients with hypertension. However, the pharmacists who conducted the interventions were highly selective. They were well respected due to their high degree qualification and work status (tertiary hospital). This would limit the potential of the results to be generalized. China has a serious shortage of clinical pharmacist workforce. It will be challenging to add further workloads on the already overloaded pharmacists.

\section{Limitations}

This study has several limitations. First, the trial ended after 6 months due to resource restrictions. It did not test the long-term effect of the interventions. Second, this study did not perform detailed costing analyses on the interventions, but we recorded an average of 14 minutes of contact time per month from the pharmacists and their assistants for each participant in the intervention group. Third, the trial could not adopt double blindness in the design. Improvement in the control group was observed in this study, albeit at a smaller scale. We could not exclude the potential flowover effect when a medical doctor serves both patients in the intervention and control groups. This may lead to an underestimation of the effect size of the interventions. Fourth, a baseline difference in medication adherence appeared between the intervention and the control groups although the 2 groups of participants were randomly allocated. This was an unexpected result possibly due to the high diversity of patients with hypertension and the limited sample size. A stratified randomization strategy may generate a more balanced sample.

\section{CONCLUSION}

The value of pharmacists may have been underappreciated. This study shows that pharmacists can make a significant contribution to improving community management of hypertension through educating patients and providing medication consultations with medical doctors, although the

\section{A systems approach is needed to maximize the role and functions of pharmacists to manage chronic diseases.}


long-term effect remains unclear. This study demonstrated that community pharmacist interventions have significant short-term effects on improving the knowledge and medication adherence of hypertensive patients, as well as timely adjustments of drug therapy regimens from medical doctors, resulting in lowered blood pressure and increased control rate. However, more and better-educated pharmacists would be needed before such an initiative can be introduced. Equally important is the improvement of the entire care process centered around the need of patients. This often requires adjustments of roles and functions of various health professionals. There is a need to increase the public and policy makers' awareness about the important role of community pharmacists in managing chronic conditions. Further studies should assess the long-term effect and cost-effectiveness of such services.

Acknowledgments: The authors are grateful for the support of all the study participants and the health institutions involved in this study.

Funding: This study was funded by the National Natural Science Foundation of China (71740018).

Author contributions: All authors contributed to writing, reviewing, and editing the article.

Competing interests: None declared.

\section{REFERENCES}

1. Chobanian AV, Bakris GL, Black HR, et al. Seventh report of the Joint National Committee on Prevention, Detection, Evaluation, and Treatment of High Blood Pressure. Hypertension. 2003;42(6):12061252. CrossRef. Medline

2. Hypertension. World Health Organization. August 25, 2021. Accessed November 16, 2021. https://www. who.int/news-room/ fact-sheets/detail/hypertension

3. Cardiovascular diseases. World Health Organization. June 11 2021. Accessed November 16, 2021. https://www.who.int/news room/fact-sheets/detail/cardiovascular-diseases-(cvds)

4. National Center for Cardiovascular Diseases, China. Report on Cardiovascular Diseases in China 2018. National Center for Cardiovascular Diseases; 2019. Accessed November 16, 2021. http://www.nccd.org.cn/News/Information/Index/1089

5. Di Palo KE, Kish T. The role of the pharmacist in hypertension management. Curr Opin Cardiol. 2018;33(4):382-387. CrossRef. Medline

6. Al-Noumani H, Wu JR, Barksdale D, Knafl G, AlKhasawneh E, Sherwood G. Health beliefs and medication adherence in Omanis with hypertension. J Cardiovasc Nurs. 2018;33(6):518-526. CrossRef. Medline

7. Abegaz TM, Shehab A, Gebreyohannes EA, Bhagavathula AS, Elnour AA. Nonadherence to antihypertensive drugs. Medicine (Baltimore). 2017;96(4):e5641. CrossRef. Medline

8. Lamb SA, Al Hamarneh YN, Houle SKD, Leung AA, Tsuyuki RT Hypertension Canada's 2017 guidelines for diagnosis, risk assessment, prevention and treatment of hypertension in adults for pharmacists: an update. Can Pharm J (OH). 2018;151(1):33-42. CrossRef. Medline

9. Morgado M, Rolo S, Castelo-Branco M. Pharmacist intervention program to enhance hypertension control: a randomised controlled trial. Int J Clin Pharm. 2011 ;33(1):132-140. CrossRef. Medline

10. Pan $F, W u H$, Liu $C$, et al. Effects of home telemonitoring on the control of high blood pressure: a randomised control trial in the Fangzhuang Community Health Center, Beijing. Aust J Prim Health. 2018;24(5):398-403. CrossRef. Medline

11. Pellegrino AN, Martin MT, Tilton JJ, Touchette DR. Medication therapy management services: definitions and outcomes. Drugs. 2009;69 (4):393-406. CrossRef. Medline

12. Zillich AJ, Sutherland JM, Kumbera PA, Carter BL. Hypertension outcomes through blood pressure monitoring and evaluation by pharmacists (HOME study). J Gen Intern Med. 2005;20(12):1091-1096. CrossRef. Medline

13. Bajorek BV, LeMay KS, Magin PJ, Roberts C, Krass I, Armour CL. Management of hypertension in an Australian community pharmacy setting - patients' beliefs and perspectives. Int J Pharm Pract. 2017;25(4):263-273. CrossRef. Medline

14. Chabot I, Moisan J, Grégoire JP, Milot A. Pharmacist intervention program for control of hypertension. Ann Pharmacother. 2003;37 (9): 1 186-1 193. CrossRef. Medline

15. Marra C, Johnston K, Santschi V, Tsuyuki RT. Cost-effectiveness of pharmacist care for managing hypertension in Canada. Can Pharm J (Ott). 2017;150(3):184-197. CrossRef. Medline

16. Okada H, Johnston K, Nakayama T, Marra C, Tsuyuki R. First world solutions: pharmacists tackling the burden of hypertension in Japan. SSRN Electronic J. Preprint posted online March 16, 2019. CrossRef

17. Fikri-Benbrahim N, Faus MJ, Martínez-Martínez F, SabaterHernández D. Impact of a community pharmacists' hypertensioncare service on medication adherence. The AFenPA study. Res Social Adm Pharm. 2013;9(6):797-805. CrossRef. Medline

18. Bajorek B, LeMay KS, Magin P, Roberts C, Krass I, Armour CL. Implementation and evaluation of a pharmacist-led hypertension management service in primary care: outcomes and methodological challenges. Pharm Pract (Granada). 2016;14(2):723-723. CrossRef. Medline

19. Reeves L, Robinson K, McClelland T, Adedoyin CA, Broeseker A, Adunlin G. Pharmacist interventions in the management of blood pressure control and adherence to antihypertensive medications: a systematic review of randomized controlled trials. J Pharm Pract. 2021;34(3):480-492. CrossRef. Medline

20. Morgado MP, Morgado SR, Mendes LC, Pereira L, Castelo-Branco $M$. Pharmacist interventions to enhance blood pressure control and adherence to antihypertensive therapy: Review and meta-analysis. Am J Health Syst Pharm. 2011 ;68(3):241-253. CrossRef. Medline

21. Government adds pharmacists into primary and community care. News release. British Columbia Government. June 5, 2018. Accessed November 16, 2021. https://news.gov.bc.ca/releases/ 2018HLTH0055-001118

22. Hirsch JD, Steers N, Adler DS, et al. Primary care-based, pharmacistphysician collaborative medication-therapy management of hypertension: a randomized, pragmatic trial. Clin Ther. 2014;36 (9): 1244-1254. CrossRef. Medline

23. Cheema $E$, Sutcliffe $P$, Singer DRJ. The impact of interventions by pharmacists in community pharmacies on control of hypertension: a systematic review and meta-analysis of randomized controlled trials. Br J Clin Pharmacol. 2014;78(6):1238-1247. CrossRef. Medline

24. Joint Committee for Guideline Revision. 2018 Chinese Guidelines for Prevention and Treatment of Hypertension-A report of the Revision Committee of Chinese Guidelines for Prevention and Treatment of Hypertension. J Geriatr Cardiol. 2019;16(3):182-241. Medline 
25. International Pharmaceutical Federation (FIP). 2012 FIP Global Pharmacy Workforce Report. FIP; 2013. Accessed November 16, 2021. https://www.fip.org/file/1414

26. Number of community health service centers. National Bureau of Statistics of China. Accessed November 16, 2021. http://www. stats.gov.cn/english/

27. Sun M, Rasooly A, Jian W. Quality of primary health care in China: an analysis of data from a nationwide longitudinal survey. Lancet. 2018;392:S74. CrossRef

28. Lu J, Lu Y, Wang X, et al. Prevalence, awareness, treatment, and control of hypertension in China: data from 1.7 million adults in a population-based screening study (China PEACE Million Persons Project). Lancet. 2017;390(101 12):2549-2558. CrossRef. Medline

29. Zhou B, Danaei G, Stevens GA, et al; NCD Risk Factor Collaboration (NCD-RisC). Long-term and recent trends in hypertension awareness, treatment, and control in 12 high-income countries: an analysis of 123 nationally representative surveys. Lancet. 2019;394 (10199):639-651. CrossRef. Medline

30. National basic public health service project brochure. National Health Commission of the People's Republic of China. Accessed November 16, 2021. https://www.nhc.gov.cn/ewebeditor/ uploadfile/2014/08/20140812080350187.pdf

31. China National Basic Public Health Service Project Basic Hypertension Management Office, Basic Hypertension Management Expert Committee. The National Guidelines for the Prevention and Control of Hypertension in Primary Medical Institutions. Chinese Circulation J. 2017;32(11):1041-1048.

32. Yingxian S. Suggestions on China mode of prevention, diagnosis and treatment of hypertension. Xiehe Med J. 2019;10(2):97-99. Accessed November 16, 2021. https://d.wanfangdata.com.cn/ periodical/xhyx201902001

33. Li X, Li T, Chen J, et al. A WeChat-based self-management intervention for community middle-aged and elderly adults with hypertension in Guangzhou, China: a cluster-randomized controlled trial. Int J Environ Res Public Health. 2019;16(21):4058. CrossRef. Medline

34. National Health and Family Planning Commission of the People's Republic of China. China Health and Family Planning Statistical Yearbook 2019. Peking Union Medical College Publishing House; 2019.

35. Li X, Lu J, Hu S, et al. The primary health-care system in China. Lancet. 2017;390(10112):2584-2594. CrossRef. Medline

36. Lei $X$, Jiang $H$, Liu C, Ferrier A, Mugavin J. Self-medication practice and associated factors among residents in Wuhan, China. Int $J$ Environ Res Public Health. 2018;15(1):68. CrossRef. Medline

37. 2019 statistical yearbook. The People's Government of Zunyi, China. Accessed November 16, 2021. http://www.zunyi.gov.cn/sjfb/ †jxx/tjni_5860125/202007/†20200711_68833237.html

38. National Bureau of Statistics of China. China Statistical Yearbook 2019. China Statistics Press; 2019

39. How are the income group thresholds determined? World Bank. Accessed November 16, 2021. https://datahelpdesk.worldbank. $\mathrm{org} / \mathrm{knowledgebase/articles/378833-how-are-the-inc}$

40. Guizhou Statistical Yearbook. 2019. Guizhou Provincial Bureau of Statistics.

41. Introduction of Huichuan district. The People's Government of Zunyi, China. Accessed November 16, 2021. http://www.zyhc.gov.cn/ zjkfq/

42. Wang Z, Chen Z, Zhang L, et al. Status of hypertension in China: results from the China Hypertension Survey, 2012-2015. Circulation. 2018;137(22):2344-2356. CrossRef. Medline

43. Notice on the issuance of China's work plan for the prevention and treatment of chronic diseases (2012-2015). National Health Commission of the People's Republic of China.
44. Faul F, Erdfelder E, Buchner A, Lang AG. Statistical power analyses using G*Power 3.1: Tests for correlation and regression analyses. Behav Res Methods. 2009;41(4):1149-1 160. CrossRef. Medline

45. Meng Q, Xu L, Zhang Y, et al. Trends in access to health services and financial protection in China between 2003 and 2011: a crosssectional study. Lancet. 2012;379(9818):805-814. CrossRef. Medline

46. Martins D, Gor D, Teklehaimanot S, Norris K. High blood pressure knowledge in an urban African-American community. Ethn Dis. $2001 ; 11(1): 90-96$. Medline

47. Morisky DE, Ang A, Krousel-Wood M, Ward HJ. Predictive validity of a medication adherence measure in an outpatient setting. J Clin Hypertens (Greenwich). 2008;10(5):348-354. CrossRef. Medline

48. World Health Organization (WHO). Guideline: Sodium Intake for Adults and Children. WHO; 2012. Accessed November 16, 2021. https://www.who.int/publications-detail/9789241504836

49. World Health Organization (WHO). Global Recommendations on Physical Activity for Health. WHO; 2010. Accessed November 16 , 2021. https://www.who.int/dietphysicalactivity/publications/ 9789241599979/en/

50. Tan ECK, Stewart K, Elliott RA, George J. Pharmacist consultations in general practice clinics: the Pharmacists in Practice Study (PIPS). Res Social Adm Pharm. 2014;10(4):623-632. CrossRef. Medline

51. Lecturer ACO, Pharmacist DUAC, Lecturer EFE. Outcomes of pharmaceutical care intervention to hypertensive patients in a Nigerian community pharmacy. Int J Pharm Pract. 2006;14(2):115-122. CrossRef

52. Hedegaard U, Kjeldsen U, Pottegård A, et al. Improving medication adherence in patients with hypertension: a randomized trial. Am J Med. 2015;128(12):1351-1361. CrossRef. Medline

53. Hazen ACM, Zwart DLM, Poldervaart JM, et al. Non-dispensing pharmacists' actions and solutions of drug therapy problems among elderly polypharmacy patients in primary care. Fam Pract. 2019;36 (5):544-551. CrossRef. Medline

54. Santschi V, Chiolero A, Colosimo AL, et al. Improving blood pressure control through pharmacist interventions: a meta-analysis of randomized controlled trials. J Am Heart Assoc. 2014;3(2):e000718. CrossRef. Medline

55. Samir Abdin M, Grenier-Gosselin L, Guénette L. Impact of pharmacists' interventions on the pharmacotherapy of patients with complex needs monitored in multidisciplinary primary care teams. Int J Pharm Pract. 2020;28(1):75-83. CrossRef. Medline

56. Amer M, Rahman N, Nazir SR, et al. Impact of pharmacist's intervention on disease related knowledge, medication adherence, HRQoL and control of blood pressure among hypertensive patients. Pak J Pharm Sci. 2018;31(6) (Supplementary):2607-2616. Medline

57. Saleem F, Hassali MA, Shafie AA, et al. Pharmacist intervention in improving hypertension-related knowledge, treatment medication adherence and health-related quality of life: a non-clinical randomized controlled trial. Health Expect. 2015;18(5):1270-1281. CrossRef. Medline

58. Zeng Z, Wang X, Wang Z, Guo R, Feng R. [Empirical research of the relationship between related knowledge, attitude and behavior of hypertension patients based on the structural equation model]. Zhong Nan Da Xue Xue Bao Yi Xue Ban. 2017;42(2):195-201. Medline

59. Shi S, Shen Z, Duan Y, Ding S, Zhong Z. Association between medication literacy and medication adherence among patients with hypertension. Front Pharmacol. 2019;10:822. CrossRef. Medline

60. Marfo AFA, Owusu-Daaku FT. Exploring the extended role of the community pharmacist in improving blood pressure control among hypertensive patients in a developing setting. J Pharm Policy Pract. 2017;10(1):39. CrossRef. Medline 
61. Muenster SJ, Carter BL, Weber CA, et al. Description of pharmacist interventions during physician-pharmacist co-management of hypertension. Pharm World Sci. 2007;30(1):128-135. CrossRef. Medline

62. Chua SS, Kok LC, Yusof FAM, et al. Pharmaceutical care issues identified by pharmacists in patients with diabetes, hypertension or hyperlipidaemia in primary care settings. BMC Health Serv Res. 2012;12(1):388. CrossRef. Medline

63. Tang C, Liu C, Fang P, Xiang Y, Min R. Work-related accumulated fatigue among doctors in tertiary hospitals: a cross-sectional survey in six provinces of China. Int J Environ Res Public Health. 2019;16 (17):3049. CrossRef. Medline

64. Sitbon M, Corny J, Beaussier H, Bézie Y. The effect of partial patients' adherence to antihypertensive drugs: scope for pharmacists' role in hypertension care. Int J Clin Pharm. 2018;40(1):1-2. CrossRef. Medline

65. Tobari H, Arimoto T, Shimojo N, et al. Physician-pharmacist cooperation program for blood pressure control in patients with hypertension: a randomized-controlled trial. Am J Hypertens. 2010;23 (10):1 144-1152. CrossRef. Medline

66. Carter BL, Rogers M, Daly J, Zheng S, James PA. The potency of team-based care interventions for hypertension: a meta-analysis. Arch Intern Med. 2009;169(19):1748-1755. CrossRef. Medline

67. Wentzlaff DM, Carter BL, Ardery G, et al. Sustained blood pressure control following discontinuation of a pharmacist intervention. J Clin Hypertens. 2011;13(6):431-437. CrossRef. Medline

68. Fikri-Benbrahim N, Faus MJ, Martínez-Martínez F, Alsina DGS, Sabater-Hernández D. Effect of a pharmacist intervention in Spanish community pharmacies on blood pressure control in hypertensive patients. Am J Health Syst Pharm. 2012;69(15):1311-1318. CrossRef. Medline

69. Stewart K, George J, Mc Namara KP, et al. A multifaceted pharmacist intervention to improve antihypertensive adherence: a clusterrandomized, controlled trial (HAPPy trial). J Clin Pharm Ther. 2014;39(5):527-534. CrossRef. Medline

70. Registered licensed pharmacist nationwide. Government of China. Certification Center for Licensed Pharmacist of NMPA. Accessed November 16, 2021. hitp://www.cqlp.org/

71. World Health Organization (WHO). World Health Statistics 2019: Monitoring Health for the SDGs, Sustainable Development Goals. Accessed November 16, 2021. https://apps.who.int/iris/handle/ $10665 / 324835$

72. Service specification for registered pharmacists. National Medical Products Administration of China. Accessed November 16, 2021. http://english.nmpa.gov.cn/

73. Opinions on accelerating the high quality development of pharmaceutical services. Government of China National Administration of Traditional Chinese Medicine. November 28, 2018. Accessed November 16, 2021. http://bgs.satcm.gov.cn/ zhengcewenjian/2018-11-28/8485.html

74. Nuño R, Coleman K, Bengoa R, Sauto R. Integrated care for chronic conditions: the contribution of the ICCC Framework. Health Policy. 2012;105(1):55-64. CrossRef. Medline

75. China's medium- and long-term plan for the prevention and treatment of chronic disease. General Office of the State Council on Printing and Distribution China's Prevention and Treatment of Chronic Diseases. Accessed November 16, 2021. http://www.gov. $\mathrm{cn} /$ _hhengce/content/2017-02/14/content_5167886.htm

\section{Peer Reviewed}

Received: August 19, 2020; Accepted: October 15, 2021.

Cite this article as: Li Y, Liu G, Liu C, et al. Effects of pharmacist intervention on community control of hypertension: a randomized controlled trial in Zunyi, China. Glob Health Sci Pract. 2021;9(4):890-904. https://doi.org/10.9745/GHSP-D-20-00505

(C) Li et al. This is an open-access article distributed under the terms of the Creative Commons Attribution 4.0 International License (CC BY 4.0), which permits unrestricted use, distribution, and reproduction in any medium, provided the original author and source are properly cited. To view a copy of the license, visit https://creativecommons.org/licenses/by/4.0/. When linking to this article, please use the following permanent link: https://doi. org/10.9745/GHSP-D-20-00505 